\title{
Pediculosis por Haematopinus quadripertusus en bovinos de Salta, Argentina. Primera descripción
}

\author{
Marin, R.E. ${ }^{\text {; }}$ Aguirre, D.H. ${ }^{2}$; Cafrune, M.M. ${ }^{2}$; Viñabal, A.E. ${ }^{2}$ \\ ${ }^{1}$ Fac. Cs. Agrarias, UNJu, Alberdi 47, 4600 Jujuy, Argentina. ${ }^{2}$ INTA IIACS Leales, \\ RN 68, Km 172, 4400 Salta, Argentina. E-mail: raulemarin@hotmail.com
}

\begin{abstract}
Resumen
Marin, R.E.; Aguirre, D.H.; Cafrune, M.M.; Viñabal, A.E.: Pediculosis por Haematopinus quadripertusus en bovinos de Salta, Argentina. Primera descripción. Rev. Vet. 29: 1, 57-60, 2018. Se describe un brote de pediculosis por Haematopinus quadripertusus, Anoplura: Haematopinidae (piojo de la cola del bovino), en un establecimiento ganadero dedicado a la cría bovina situado en la región chaqueña de la Provincia de Salta, Argentina. El cuadro clínico ocurrió en solo uno de los cuatro rodeos de hembras y también en la tropa de toros, con severa infestación de la punta de la cola y la región perineal, principalmente alrededor de la vulva y el ano, al igual que en los bordes palpebrales superior e inferior. La región de la base de la cola y la zona perineal de los vacunos afectados mostraron dermatitis pruriginosa severa a moderada, con alopecia leve. La prevalencia de la pediculosis se estimó en $70 \%$ para las hembras y $35 \%$ para los toros. El rodeo infestado por H. quadripertusus presentó una tasa de preñez más baja que los rodeos restantes. Esta publicación constituye el primer informe de $H$. quadripertusus en nuestro país, completando la lista de haematopínidos con potencial parasitario para los bovinos de Argentina.
\end{abstract}

Palabras clave: bovino, Haematopinus quadripertusus, piojo de la cola, Salta, Argentina.

\begin{abstract}
Marin, R.E.; Aguirre, D.H.; Cafrune, M.M.; Viñabal, A.E.: Pediculosis by Haematopinus quadripertusus in cattle from Salta, Argentina. First description. Rev. Vet. 29: 1, 57-60, 2018. An outbreak of pediculosis by Haematopinus quadripertusus, Anoplura: Haematopinidae (bovine tail louse) is described in a cattle breeding establishment located in the Chaco region of Salta Province, Argentina. The clinical signs occurred in only one of four cow herds and also in the bull's herd, with severe infestation of the tail tip and the perineal region, mainly around the vulva and the anus, as well as in the upper and lower palpebral borders. The regions of caudal basis and perineal of the affected animals showed severe to moderate pruritic dermatitis with mild alopecia. Prevalence of pediculosis was estimated at $70 \%$ in the females and about $35 \%$ in the bulls. The herd infested by H. quadripertusus had a lower pregnancy rate than the remainder herds. This is the first report of $H$. quadripertusus in our country, which complete the list of Haematopinidae with parasitic potential for cattle in Argentina.
\end{abstract}

Key words: cattle, Haematopinus quadripertusus, tail louse, Salta, Argentina.

\section{INTRODUCCIÓN}

Los procesos de expansión e intensificación de la ganadería bovina en los diferentes ecosistemas del noroeste argentino, plantean la necesidad de reconocer y controlar los problemas sanitarios que impactan sobre dicho ganado. Entre ellos se ubican los ectoparásitos que, como en el caso de los piojos, inciden negativamente en la producción bovina ${ }^{6,11}$.

Recibido: 27 junio 2017 / Aceptado: 24 agosto 2017

Presentado en XX Reun.Cient.Técn.Asoc.Arg.Vet.Lab.

Diagnóst.,Tucumán, Argentina., 2014
Las primeras citas sobre presencia de piojos en vacunos de nuestro país datan de hace poco más de un siglo $^{6}$. Más tarde, los estudios de Lahille y Roveda aportaron abundante información sobre esta parasitosis en el ganado local, pero esos trabajos se discontinuaron, salvo contadas excepciones. Tres especies de piojos picadores son reconocidas hasta hoy como parásitos de los bovinos en Argentina: Haematopinus eurysternus, Linognathus vituliy y Solenopotes capillatus, a los que se suma una cuarta especie masticadora: Bovicola bovis ${ }^{1,5}$.

La familia Haematopinidae incluye a los piojos de mayor tamaño de los animales domésticos. Comprende 
un solo género, Haematopinus, cuyas especies son todas de importancia por su acción expoliativa e irritante. Los bovinos son parasitados por tres especies del género: H. eurysternus, $H$. quadripertusus y $H$. tuberculatus, aunque este último es más conocido como parásito de los búfalos ${ }^{4}$.

Se reconoce que una elevada parasitosis por Haematopinus sp puede ser causa de anemia, predisponiendo a enfermedades respiratorias, aborto o muerte ${ }^{12}$, además de intenso prurito, generando lesiones traumáticas auto-infligidas y pérdidas productivas.

En este trabajo se describe por primera vez en Argentina, la ocurrencia clínica de un brote de H. quadripertusus en un rodeo bovino de la región chaqueña de la Provincia de Salta.

\section{MATERIAL Y MÉTODOS}

El brote se presentó en un establecimiento de 5.500 ha de extensión, dedicado a la ganadería de cría bovina, ubicado al extremo este del Departamento Anta

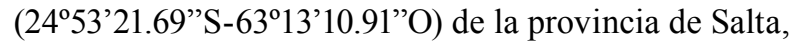
dentro la región fitogeográfica chaqueña, área con una pluviometría anual de $750 \mathrm{~mm}$. El rodeo se componía por 2.070 vientres cruza índica con predominio de razas Braford y Brangus negro y colorado, separados en cuatro grupos con servicio por monta natural.

Durante sendos exámenes para diagnóstico de gestación, en mayo y junio de 2014, se detectó una parasitación por piojos ubicados casi exclusivamente en las zonas caudal y perineal de las hembras bovinas de uno de los rodeos. En la segunda ocasión (junio) este rodeo se inspeccionó clínicamente, recolectándose ejemplares de piojos que ingresaron al laboratorio donde, después de ser aclarados con lactofenol, fueron examinados microscópicamente.

La determinación taxonómica se realizó siguiendo el trabajo de comparación de especies de Haematopi$n u s$ en bovinos de Meleney y Kim ${ }^{3}$. Con posterioridad, durante el examen clínico anual de los toros, también se observó en ellos la infestación por piojos. El rodeo problema fue tratado con un endectocida inyectable de larga acción (Ivomec Gold 3.15 Merial) en dosis única de $1 \mathrm{~cm}^{3} / 50 \mathrm{~kg}$ de peso.

En los rodeos restantes solo se realizó vigilancia epidemiológica, examinándolos periódicamente para establecer la presencia del ectoparásito. Esta práctica se extendió al ciclo productivo posterior (año 2015).

\section{RESULTADOS}

Clínicamente los animales afectados presentaron dermatitis pruriginosa moderada a severa, con alopecia moderada en forma difusa en la región de la base de la cola y perineal, tanto en las hembras como en los machos (Figura 1).

Las hembras mostraban una infestación por piojos de grado leve, moderado o severo alrededor de la vulva (Figura 2), mientras que en los machos los parásitos se

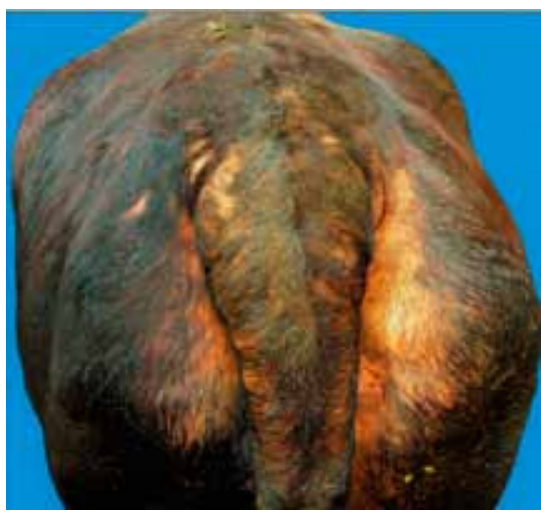

Figura 1. Dermatitis pruriginosa difusa severa con alopecia y presencia de $H$. quadripertusus.



Figura 2. Infestación grave por H. quadripertusus en los labios vulvares

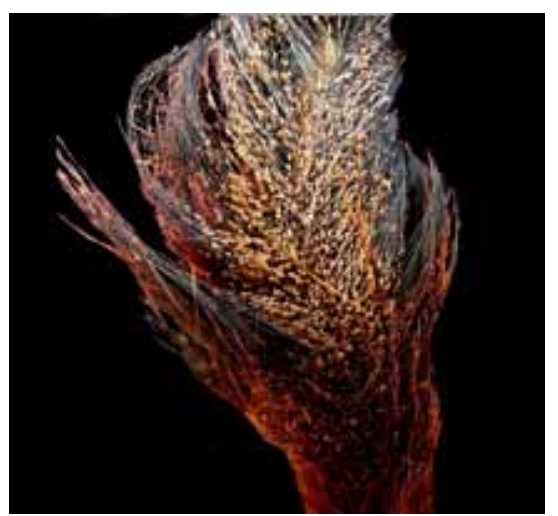

Figura 3. Intensa infestación por liendres de H. quadripertusus en el extremo caudal de un bovino.

establecían alrededor del ano, zona de piel más delgada, propicia para la fijación de los estadios ninfales de los piojos.

La cara inferior de la cola, desde el extremo distal hasta su inserción dorsal, se reveló como zona de tránsito de los parásitos, mientras que la presencia de huevos o liendres era más abundante en el extremo caudal (Figura 3), detectándose también en ciertos casos en los pelos de la comisura labial de la vulva.

Algunos animales mostraban completa depilación de la punta de la cola, posiblemente debido a traumatismo autoinfligido como consecuencia del prurito. Se observó además una infestación moderada en los bordes 


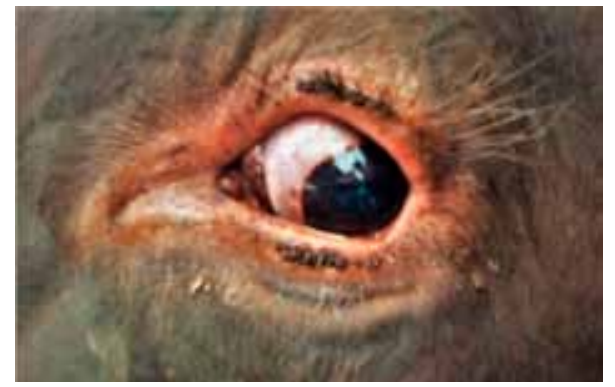

Figura 4. Infestación por $H$. quadripertusus en los bordes palpebrales de un bovino.

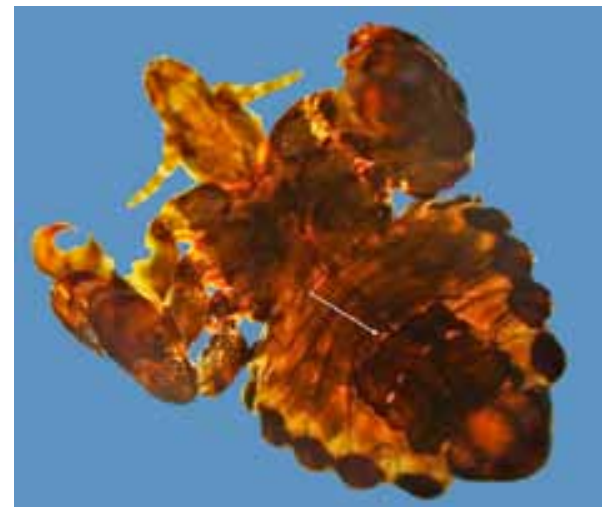

Figura 5. Macho adulto de H. quadripertusus mostrando la placa ventral (carácter taxonómico).

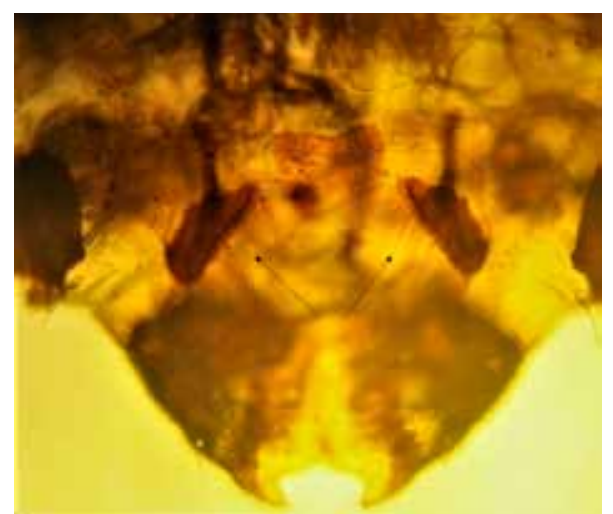

Figura 6. Porción distal de una hembra adulta de $H$. quadripertusus, mostrando la genitalia (carácter taxonómico).

palpebrales de los toros, pero sin evidencias de efectos clínicos de consideración (Figura 4).

El examen de los piojos adultos permitió establecer que se trataba de ejemplares de Haematopinus quadripertusus Fahrenhölz 1916 (Figuras 5 y 6), conocidos vulgarmente como piojo de la cola de los bovinos, región anatómica en la cual prevalecían al momento de ser colectados

Curiosamente los piojos parasitaron a uno solo de los cuatro rodeos con que contaba el establecimiento ganadero. La prevalencia de la pediculosis fue de alrededor de un $70 \%$ en el rodeo de hembras afectadas, mientras que en los toros fue cercano al $35 \%$, incidiendo más en los bovinos de capa clara (marrón) que en los de capa oscura (negra).
Al momento del examen clínico y reproductivo anual de los toros, éstos ya integraban un único rodeo después de haber sido asignados por grupos al servicio en los distintos lotes de vacas, sin que pudiera establecerse cuales habían trabajado en el rodeo infestado. Por otra parte, en 2014, el porcentaje general de preñez para la totalidad de vientres del establecimiento fue de $86 \%$. Pero mientras los tres rodeos no afectados por H. quadripertusus lograron en conjunto un $89,5 \%$ de preñez, el rodeo con pediculosis, compuesto principalmente por vaquillonas y algunas vacas CUT (279 vientres), alcanzó apenas un $66 \%$ de preñez.

El tratamiento antiparasitario aplicado fue efectivo para el control de H. quadripertusus, el cual no volvió a aparecer durante el siguiente ciclo productivo. En ninguna oportunidad se observaron otras especies de piojos en los bovinos del establecimiento.

\section{DISCUSIÓN}

En Argentina hay escasos registros sobre la situación de la pediculosis de los bovinos. Una de las últimas publicaciones relevantes sobre el particular fue la actualización de Prieto y colaboradores (1991) ${ }^{6}$. Un estudio posterior mediante encuestas a productores ganaderos del este de la provincia de La Pampa reveló que el $43 \%$ habían tenido piojos en sus vacunos, sobre todo en invierno, y más de la mitad de ellos aplicaban al menos dos tratamientos anuales para controlar o prevenir la parasitosis ${ }^{11}$.

En cuanto a $H$. quadripertusus, su presencia fue considerada como "muy probable" en Argentina hacia fines del siglo pasado ${ }^{1}$, pero no había sido confirmada hasta ahora. Según Roberts, los estudios de Fahrenhölz (1916) describieron a H. quadripertusus a partir de ejemplares colectados en Camerún, África ${ }^{7}$. El nombre de esta especie fue más tarde relegado a una sinonimia de H. eurysternus, pero en 1947 se revalidó en base al estudio de especímenes recogidos de la cola de bovinos de Florida, USA.

Finalmente, en 1974 Meleney y Kim revisaron las especies de Haematopinus de los vacunos, redescribiendo a $H$. quadripertusus en base de sus rasgos morfológicos, distribución geográfica y biotipos de hospedadores y sitios (microhábitats) predilectos para su fijación ${ }^{3}$.

En efecto, las dos especies de Haematopinus más asociadas con los bovinos muestran diferencias en todos los aspectos mencionados ${ }^{3}$. Mientras $H$. eurysternus es más frecuente en ambientes templados, $H$. quadripertusus se halla habitualmente en ambientes tropicales y subtropicales.

De hecho, y aparte los sitios ya citados, fue diagnosticado en México, Costa Rica, Panamá, Libia, Israel, India, Australia y Pakistán ${ }^{8,10,13}$, país este último en el que también se encontró sobre búfalos ${ }^{8}$. En Sudamérica, $H$. quadripertusus fue informado en Venezuela ${ }^{3}$ y Brasil, aunque en este último hasta ahora se halló solamente en tres Estados: Minas Gerais, Sergipe y Pará ${ }^{10}$. 
Respecto a los biotipos bovinos preferidos, H. eurysternus parasita sobre todo a Bos taurus domésticos, en tanto $H$. quadripertusus infesta con mayor frecuencia a Bos indicus y sus cruzas, aunque también se encontró en ganado de origen europeo ${ }^{4,6}$.

Los adultos de H. quadripertusus se confinan generalmente en la porción distal y más pilosa de la cola, o alrededor de los ojos y orejas. Los huevos son depositados casi exclusivamente en el extremo caudal -y tras su eclosión- las ninfas migran a las regiones periféricas del ano, vulva y ojos, donde la piel más delgada facilita la hematofagia.

Por último, los principales rasgos morfológicos para el diagnóstico de $H$. quadripertusus se centran en la forma de la placa esternal, tanto en hembras como en machos, así como la forma y quetotaxia de la genitalia de hembras y machos y de la placa subgenital de los machos ${ }^{3}$.

La prevalencia parasitaria del caso actual fue intermedia respecto a otros registros americanos en rodeos más pequeños ( $<100$ cabezas), los cuales oscilaron entre $21,3 \%$ en México ${ }^{8}$ y $100 \%$ en Brasil ${ }^{10}$. Coincidiendo con la mayoría de informes previos ${ }^{8,10,13}$, H. quadripertusus se presentó en nuestro caso como infestación pura, si bien existen informes de su ocurrencia concomitante con otras especies de piojos, como L. vituli ${ }^{2}$.

El mayor tamaño de H. quadripertusus (hembras = $4,5 \mathrm{~mm}$ ) califica a esta especie como más nociva que $H$. eurysternus (hembras $=3,5 \mathrm{~mm}$ ). Por otro lado, H. quadripertusus no muestra variación estacional marcada en su acción parasitaria, a diferencia de otras especies de piojos bovinos, más activas durante el invierno ${ }^{11,13}$.

En algunos países, como Israel, las pediculosis por H. quadripertusus son más relevantes que las causadas por H. eurysternus y capaces de generar patologías asociadas, como queratoconjuntivis y papilomatosis periorbital ${ }^{13}$. Por otra parte, no se descarta que la infestación por piojos hematófagos contribuya a la transmisión de hemoparásitos, como Anaplasma marginale ${ }^{9,14}$.

Si bien es común que las pediculosis se asocien con ganado joven estresado y desnutrido, ocurren excepciones a esta regla, como las reportadas en México ${ }^{8} \mathrm{y}$ Brasil ${ }^{10}$, donde resultaron parasitados bovinos adultos con buena condición corporal, al igual que los del caso aquí descrito.

El presente hallazgo de $H$. quadripertusus completa la lista de haematopínidos con potencial parasitario para los bovinos de Argentina, sobre todo en los biotipos índicos de áreas tropicales y subtropicales, susceptibles tanto a esta especie como a $H$. tuberculatus. Si bien este último todavía no fue informado en vacunos de nuestro país, su presencia en búfalos locales ${ }^{4}$ hace presumir la posibilidad de hallarlos también en bovinos, particularmente en aquéllos que comparten zonas de pastoreo con búfalos ${ }^{3}$.

\section{REFERENCIAS}

1. Castro D, Cicchino AC. 1998. Anoplura. En: Biodiversidad de artrópodos argentinos. Una perspectiva biotaxonómica (Morrone J., Coscarón S., Ed.), Ediciones Sur, La Plata, Argentina, p. 125-139.

2. Gabaj MM, Beesley WN, Awan MA. 1993. Lice of farm animals in Libya. Med Vet Entomol 7: 138-140.

3. Meleney WP, Kim KC. 1974. A comparative study of cattle-infesting Haematopinus, with redescription of $H$. quadripertusus (Fahrenholz, 1916) (Anoplura: Haematopinidae). J Parasitol 60: 507-522.

4. Moriena RA, Racioppi O, Santa Cruz AM, Martínez FA, Lombardero OJ. 1982. Presencia de Haematopinus tuberculatus (Burmeister, 1839) en Bubalus bubalis (Búfalo de la India) en la Provincia de Corrientes. Gaceta Vet 44: 1223-1225.

5. Olaechea F, Romero J, Prieto O, Larroza M. 2103. Ectoparásitos permanentes del ganado en Argentina. En: Enfermedades parasitarias con importancia clínica y productiva en rumiantes (Nari A, Fiel C, Eds), Editorial Hemisferio Sur, Buenos Aires, p. 485-515.

6. Prieto OH, Cicchino AC, Abrahamovich AH, Núñez JL. 1991. Piojos (Phthiraptera) parásitos del bovino y porcino. Estado actual de su conocimiento y propuestas para su manejo y control. Rev Med Vet 72: 154-174; 240-256; 264-282.

7. Roberts FH. 1950. The tail-switch louse of cattle, Haematopinus quadripertusus Fahrenholz. Aust Vet J 26: 136-138.

8. Rodríguez RI, Ojeda M, Martínez IT, Rosado JA, Quintero MT. 2016. Infestación de bovinos con Haematopinus quadripertusus en Yucatán, México. Bioagrociencias 9: 23-32.

9. Schafer A, Samia L, Stumpfs JD, Tonin AA, Moura L, Nunes D. 2013. Lice outbreak in buffaloes: Evidence of Anaplasma marginale transmission by sucking lice Haematopinus tuberculatus. J Parasitol 99: 546-547.

10. Scofield A, Ferreira K, Melo AM, Sousa CH, Barbosa JD, Góes G. 2012. Infestation by Haematopinus quadripertusus on cattle in Sao Domingos do Capim, state of Pará, Brazil. Rev Bras Parasitol Vet 21: 315-318.

11. Suárez VH et al. 2006. Prevalencia y control de los principales ectoparásitos bovinos en el este de la provincia de La Pampa, Argentina. Rev Invest Agropec (RIA, INTA) 35: 43-66.

12. Wall R, Shearer D. 2001. Lice (Phthiraptera). In: Veterinary Ectoparasites: Biology, Pathology and Control, 2nd Ed., Blackwell Science, Oxford, p.162-178.

13 Yeruham I, Hadani A, Perl S, Elad D. 2001. Keratoconjuctivitis and periorbital papillomatosis associated with heavy periorbital infestation by the tail louse Haematopinus quadripertusus in heifers. J Vet Med B 48: 133-136.

14. Zajac A, Conboy GA. 2012. Lice (Orders Anoplura and Mallophaga). In: Veterinary Clinical Parasitology, $8^{\circ}$ Ed., John Wiley \& Sons, New Jersey, p. 266-279. 